\section{Coli and Coliphage}

Experiments in Molecular Genetics. By Jeffrey H. Miller. Pp. xv+466. (Cold Spring Harbor Laboratory: Cold Spring Harbor, New York, 1972.)

MANY of the exciting advances in modern biology were dependent on the study of genetic variants: genetics has become a very powerful biochemical tool. Because of its pre-eminent suitability for both genetical and biochemical investigation, Escherichia coli undoubtedly remains the organism of choice for the elucidation of many fundamental biological principles. Jeffrey Miller's laboratory manual, growing out of the famous bacterial genetics course at Cold Spring Harbor, deals exclusively and comprehensively with the molecular genetics of $E$. coli and its phages.

The presentation begins at the simplest level by introducing basic techniques such as dilution methods, sterile plating and viable cell counts. The subject matter gradually increases in complexity, finally covering such recent experiments as the purification and properties of repressor proteins and cellfree enzyme synthesis. Each of the nine major sections is introduced with a wellreferenced discussion of the background theory and general value of a particular experimental approach, and ends with a series of questions intended to promote further thinking. The accounts of the many individual experiments are preceded by short discussions of the rationale and strategy. The very detailed experimental protocols include lists of chemicals and apparatus required for each exercise.

One of the many strengths of the book is the profusion of advice on such matters, for example, as the choice of starting strain, the idiosyncrasies of particular mutants or the action to take on spillage of a noxious chemical.

Jeffrey Miller has succeeded in producing a superb laboratory manual, which reveals much of the lore of $E$. coli genetics. The volume should obviously find constant use in bacterial genetics laboratories for many years. The strain lists obtainable with the manual will be particularly useful to teachers who do not have access to a good strain collection.

W. J. Brammar

\section{Cybernetics}

Fundamentals of Cybernetics. By A. Ya. Lerner. Translated from Russian by E. Gros. Translation edited by F. H. George. Pp. $x+294$. (Chapman and Hall: London, August 1972.) $£ 6$.

THIS book originally appeared in the USSR as long ago as 1967: it therefore, de facto, cannot-as asserted by the editor of this English translation-provide "a good insight into the contemporary Russian approach to cybernetics". In fact, the whole face of Soviet cybernetics has changed radically since the original publication of this book, principally as a result of the efforts of the outstanding groups of cyberneticians working at the Institute of Control Sciences in Moscow and at the Institute of Cybernetics in Kiev. Even allowing for this fact, however, it is unlikely that this book will be very useful for its intended readership: for, in writing this book, Professor Lerner "wished particularly to assist people with inadequate mathematical knowledge and without the necessary time to study the works of Wiener, Shannon, Andronov, Kolmogorov and others, but who, in spite of this, wanted to study the methods of solving cybernetic problems". It is hard to see how such people will benefit in this way by reading the nineteen very brief chapters of the book which contain cursory discussions of such vast topics as automatic control, optimal control, automata, computers, adaptation, games, learning, large systems, operational control, the brain, organized systems, man and machine, and the future prospects of cybernetics. Indeed, it is ironical that the text which still provides the best introduction to the fundamentals of cybernetics is advertised on the jacket of Professor Lerner's book: this, of course, is An Introduction to Cybernetics by W. Ross Ashby whichas Stafford Beer has remarked-"is an education in itself (which) demonstrates that you can change your life quietly as well as dramatically". Brian PORTER

\section{Organic Atlas}

Atlas of Spectral Data and Physical Constants for Organic Compounds. Edited by Jeanette G. Grasselli. Pp. 1,697. (CRC Press: Cleveland, Ohio; Blackwell: Oxford, 1973.) £50.

THE Chemical Rubber Company's Handbooks of Chemistry and Physics $(H B C P)$, now in their fifty-third edition, are familiar and most useful pieces of laboratory equipment. In the past few years sixteen other Handbooks, three Manuals, a set of Tables, and now this Atlas, have appeared under the same imprint.

The volume is organized in three sections. Section $\mathrm{A}$ is introductory, dealing under separate headings with the four spectroscopic techniques; infrared, ultraviolet, nuclear magnetic resonance, and mass. Much of the material, including useful infrared correlation charts, is reprinted from $H B C P$ and will doubtless appear again in the forthcoming Handbook of Spectroscopy.
Section B contains the "Master Data Table", preceded by a synonym directory ; in spite of the title "Atlas" the data are all in tabulated numerical form. About 8,000 compounds are listed; the large majority of these appeared in $H B C P$ (fifty-second edition) and they are tabulated similarly here, with the additional spectra data and the Wiswesser line notation (WLN) code. All the spectroscopic data, with the exception of some of the ultraviolet, come from published collections. There are infrared data for about 5,550 compounds, of which almost two-thirds come from the Sadtler collections, much of the rest from the Coblentz Society (also Sadtler), and a few from the DMS, API and NBS collections. Compounds for which ultraviolet data are given number about 4,800. Data for about half of these appeared without references to source in $H B C P$ and are listed again, $\log \epsilon$ values having been converted, or reconverted, to $\epsilon$ values, and remain without references. As in $H B C P$, the so-called references in the first column are to Beilstein or Elsevier and are therefore of little use. Of the remaining ultraviolet data, there are literature references for about 450 compounds, and the rest, about 1,950, are from the Sadtler collection. The NMR data are the least comprehensive; of less than 2,600 compounds Sadtler contribute data for 2,250 , the rest being Varian, and the absence of any assignments diminishes considerably the value of this material. Of 3,050 mass spectra, 870 are Aldermaston and the rest are from the Wiley collections.

Section C comprises the indexes. After formula and physical constant (m.p. and b.p.) indexes, a large subsection is devoted to the WLN system, including an extensive permuted index designed to correlate data from compounds with common functional groups or substructures. Then follow comprehensive indexes for each of the four spectroscopic techniques, based upon wavelengths (frequencies, chemical shifts, mass numbers) and intensities of the most prominent features. Except for the ultraviolet index these also list WLN codes and their permuted abbreviations which are called, somewhat grotesquely, "Eyeball".

The WLN index system works surprisingly well even for somebody quite unfamiliar with it; it is easy to pick out, for example, all the phenyl ethers or all the $\alpha$-ketoesters listed, and, before referring to the Master Data Table, to see at a glance which of these have, say, infrared or mass spectra. The value of the Tables which list bands in order of wavelength is less certain. They are too vulnerable to the effects of converting rounded-off 